Die norwegische Studie hatte 2 Ergebnisse: TTT ist in der klinischen Routine praktikabel und eine aggressivere Vorgehensweise war mit mehr ACR/EULARBoolean-Remissionen assoziiert.

Die Arbeitsgruppe verglich die Effektivität einer strengen und einer weniger strengen TTT-Strategie anhand von 2 Studien mit Patienten, die eine Früharthritis und bislang keine krankheitsmodifizierenden Medikamente (DMARD) bekommen hatten. In ARCTIC (a randomised trial examining the benefit of ultrasound in a Clinical Tight Control regimen; $n=189$ ) lagen Remissionen bei einem DAS44 < 1,6 plus fehlende Krankheitsaktivität im Doppler-Ultraschall vor. Das Follow-up betrug 2 Jahre mit Kontrollen nach 1, 2, 3, 4, 6, 8, 10, 12, 14, 16, 20 und 24 Monaten. Die Therapie begann mit Methotrexat und Prednisolon (ausschleichend). Bei nicht ausreichender Effektivität waren nach einem festen Algorithmus erhöhtes Methotrexat, synthetische DMARD-TripleTherapie und biologische DMARD vorgesehen. NOR-VEAC (Norwegian Early Arthritis Clinic; $n=330$ ) war eine Beobachtungsstudie mit dem Ziel DAS28 <2,6. Das Monitoring erfolgte nach 1, 2 und 3 Monaten sowie vierteljährlich bis Monat 24 und halbjährlich bis Jahr 5 . Ein definierter Therapiealgorithmus lag nicht vor. Auch in NOR-VEAC erhielten die Erkrankten initial Methotrexat. Die Behandlungseskalationen erfolgten nach ungünstigen Prognosekriterien, schwerer Krankheitsaktivität und bei frühen Erosionen. Die Remissionskalkulation (ACR/EULAR-Boolean) führten die Wissenschaftler mit einem 28-Gelenk-Score durch.

\section{Treat-to-Target-Strategien mit hohen Remissionsraten}

\author{
Norvang $V$ et al. Achievement of Remission in \\ Two Early Rheumatoid Arthritis Cohorts \\ Implementing Different Treat-to-Target \\ Strategies. Arthritis Rheumatol 2020; 72: \\ 1072-1081. doi:10.1001/art.41232
}

Bei der rheumatoiden Arthritis ist das Therapieziel nicht mehr die bloße Symptomlinderung, sondern eine möglichst weitgehende Remission. Die Treat-toTarget-Strategie (TTT) schließt die Definition des gewünschten Behandlungsergebnisses, Festlegung von Therapieeskalationen und des Monitorings ein.
Die Verteilung der initialen Kovariablen war in beiden Kohorten ausgeglichen (standardisierte Differenz < 10\%). Durchschnittlich erfolgten in 2 Jahren 13 (ARCTIC) und 8 (NOR-VEAC) Verlaufskontrollen. Das mittlere Untersuchungsintervall lag im 1. Jahr bei 1,5 und 2,4 Monaten, im 2. Jahr bei 3 und 4 Monaten. Die Zielremission wurde in beiden Kohorten langfristig von etwa 2 Drittel der Patienten erreicht. Die Häufigkeiten betrugen in ARCTIC und NOR-VEAC:

- 3 Monate 37,4 vs. 45,5\%,

- 6 Monate 53,3 vs. 50,9\%,

- 12 Monate 69,8 vs. $61,0 \%$ und

- 24 Monate 66,3 vs. $65,1 \%$. 
Allerdings unterschieden sich die Erfolge in ARCTIC und NOR-VEAC hinsichtlich der ARC/EULAR-Boolean-Remissionen signifikant:

- 3 Monate 28 vs. $19,4 \%$,

- 6 Monate 32,4 vs. $25,7 \%$,

- 12 Monate 45,4 vs. $30,1 \%$ und

- 24 Monate 51,5 und 34,7\%.

Daraus resultierte nach 3, 12 und 24 Monaten ein Chancenverhältnis von OR 1,73 (95\%-Konfidenzintervall KI 1,03-2,89), OR $1,97(95 \%-K I) 1,21-3,20)$ und OR 1,82 (95\%-KI 1,05-3,16). In ARCTIC traten 529 und in NOR-VEAC 379 behandlungsassoziierte Nebenwirkungen auf. Ein TherapieSwitch erfolgte in beiden Gruppen vergleichbar häufig.

FAZIT

Die TTT-Strategie war in der Beobachtungsstudie wie in der randomisierten Studie mit hohen Remissionsraten assoziiert. Stringentere Verlaufskontrollen und ein höheres Therapieziel resultierten aber in erfolgreicheren Remissionen nach den ACR/EULAR-Kriterien. Die Autoren führen dies auf häufigere Therapieeskalationen, den Behandlungsalgorithmus, die DAS44 als Beurteilungsinstrument und das intensivere Monitoring in ARCTIC zurück. Die häufigeren Nebenwirkungen könnten auch durch die zahlreicheren Follow-Ups bedingt sein.

Dr. Susanne Krome, Melle 